\section{ITAS NEWS}

\section{Bundestag beauftragt ITAS erneut mit der Betreibung des TAB}

Auch in den kommenden Jahren wird ITAS das Büro für Technikfolgen-Abschätzung beim Deutschen Bundestag (TAB) betreiben. Das hat der Ausschuss für Bildung, Forschung und Technikfolgenabschätzung des Bundestages in seiner Sitzung am 27. Februar 2013 einstimmig beschlossen. Alle Fraktionen waren sich einig, dass die Arbeit des TAB für den Deutschen Bundestag einen hohen Stellenwert besitzt. Über den Betreiber des TAB entscheidet der Bundestag alle fünf Jahre neu. Seit Gründung des TAB im Jahr 1990 hat der Bundestag stets ITAS diese Aufgabe übertragen.

Das TAB ist eine selbständige wissenschaftliche Einrichtung, die den Deutschen Bundestag und seine Ausschüsse in Fragen des wissenschaftlich-technischen Wandels berät. Zu den Aufgaben gehören die Konzeption und Umsetzung von Projekten der Technikfolgenabschätzung, die Beobachtung und Analyse wissenschaftlichtechnischer Trends und damit zusammenhängender gesellschaftlicher Entwicklungen sowie Untersuchungen zum Innovationsgeschehen. Ziel dabei ist, die gesellschaftlichen, wirtschaftlichen und ökologischen Chancen und Risiken neuer Entwicklungen auszuloten, um so Handlungsoptionen für politische Entscheidungsträger zu entwickeln. Damit verbessert das TAB die Informationslage des Deutschen Bundestages und trägt zur wissenschaftlich fundierten Meinungsbildung und Entscheidungsfindung bei.

Unterstützt wird das TAB künftig von drei Kooperationspartnern: dem Helmholtz-Institut für Umweltforschung (Leipzig), dem Institut für Zukunftsstudien und Technologiebewertung (Berlin) sowie dem VDI/VDE-IT (VDI/VDE Innovation + Technik GmbH, Berlin).

\section{Neues Projekt gestartet: TRANSFORUM}

Das Ziel des EU-Projektes TRANSFORuM lautet: Umsetzung der im europäischen „Weißbuch zum Verkehr" genannten Wünsche in die Praxis. $\mathrm{Zu}$ diesen gewünschten Zielen gehören neben einem sauberen Stadtverkehr und einer $\mathrm{CO}_{2}$-freien Stadtlogistik die Verlagerung des Straßengüterverkehrs auf Eisenbahn- und Schiffsverkehr, die Vollendung eines europäischen Hochgeschwindigkeitsschienennetzes und der Erhalt eines dichten Schienennetzes sowie die Errichtung eines europäischen multimodalen Verkehrsinformations-, Management- und Zahlsystems. ITAS ist einer von elf Projektpartnern aus neun europäischen Ländern.

Das Projekt wird für alle wichtigen Akteure in diesem Themenfeld ein zentrales Diskussionsforum bieten. Im Rahmen von Konferenzen und thematisch auf die Weißbuchziele fokussierten Arbeitsgruppen werden sich die Akteure in einem zielorientierten Diskurs insbesondere mit den Themen Forschung und Innovation auseinandersetzen. TRANSFORuM wird politische Handlungsempfehlungen und Roadmaps erarbeiten, welche auf die konkrete Umsetzung zielen und dabei explizit die Erkenntnisse und Einschätzungen unterschiedlicher Stakeholder einbeziehen. Durch Veröffentlichungen und gezielte Kommunikation der Projektergebnisse soll die Umsetzung der Weißbuchziele zusätzlich unterstützt werden. ITAS wird sich in diesem Projekt auf die Entwicklung der Roadmaps konzentrieren und zusammen mit den Stakeholdern und dem Projektteam zielgerichtete Empfehlungen erarbeiten, wie die Weißbuchziele schrittweise umgesetzt werden können. In Workshops werden die Stakeholder ihre Problemwahrnehmungen diskutieren und in einem iterativen Prozess an der Ausarbeitung der Roadmaps mitwirken. Auf deren Basis wird ITAS schließlich konkrete Empfehlungen zur Erreichung der Ziele geben und einen weitergehenden Ausblick auf die Zukunft des europäischen Verkehrssystems liefern.

\section{Kontakt}

Jens Schippl, ITAS

Tel.: +49 (0) 721 / 608 - 23994

E-Mail: jens.schippl@kit.edu 


\section{Synthetische Biologie in der HGF: ITAS übernimmt die Begleitforschung}

Die Herstellung von neuartigen biologischen Systemen, Funktionen und Organismen, die in der Natur nicht vorkommen, gehört zu den Zielen der Synthetischen Biologie. Diese stellt (bisher) weniger ein streng abgegrenztes Forschungsfeld dar, sondern kann eher als ein Ansatz beschrieben werden, der von ingenieurswissenschaftlichen Vorstellungen geprägt ist, Lebensformen in exakt vorhersehbarer Weise zu verändern oder gar neu zu entwerfen. Außerdem bezieht die Synthetische Biologie oft verschiedene Disziplinen, wie Molekular- und Systembiologie, Chemie, (Bio-) Physik oder das computergestütze Modellieren ein. Parallel zu den großen Chancen, die das Feld für die Lösung zentraler Probleme in Bereichen wie Energie, Gesundheit und Umwelt bietet, erleben wir national wie international vermehrt akademische und öffentliche Diskussionen über potenzielle Risiken. Diese beziehen sich auf den Schutz vor Missbrauch („,biosecurity“), auf mögliche Gefahren für die menschliche Gesundheit und die Umwelt (,,biosafety“) sowie auf sozioökonomische Risiken mancher Anwendungen. Darüber hinaus sind mögliche Auswirkungen der Synthetischen Biologie auf traditionelle Vorstellungen von Leben Teil dieser Diskussionen.

Mit der im September 2012 angelaufenen „Helmholtz-Initiative Synthetische Biologie“, an der auch ITAS beteiligt ist, möchte die HelmholtzGemeinschaft (HGF) die Synthetische Biologie als ein aufstrebendes Forschungsfeld fördern und als Technologie mit hohem Anwendungspotenzial in Deutschland etablieren. Die Initiative wird vom Deutschen Krebsforschungszentrum in Heidelberg koordiniert und umfasst fünf HelmholtzZentren sowie die Universitäten Freiburg und Heidelberg. In mehreren Technologieplattformen der Initiative werden molekulare Bausteine und Schaltkreise entwickelt, die in interdisziplinären Anwendungsprojekten aus den Forschungsbereichen Gesundheit und Schlüsseltechnologien eingesetzt werden sollen. Das ITAS übernimmt im Projekt SynGovernance die Begleitforschung der Initiative. SynGovernance befasst sich speziell mit den ethischen und sozialen Aspekten der
Synthetischen Biologie sowie mit Fragen der Forschungspolitik und Förderstrategien.

\section{Kontakt}

Dr. Harald König, ITAS

Tel.: +49 (0) 721 / 608 - 23293

E-Mail: h.koenig@kit.edu

\section{$\ll 》$}

\section{Armin Grunwald in das „Deutsche Komitee für Nachhaltigkeitsfor- schung in Future Earth" berufen}

\begin{abstract}
Armin Grunwald wurde in das, von der Deutschen Forschungsgemeinschaft eingerichtete „Deutsche Komitee für Nachhaltigkeitsforschung in Future Earth" berufen. Das Komitee ist ein wissenschaftliches Beratungsgremium für nationale und internationale Aktivitäten im Rahmen der Initiative „Future Earth: research for global sustainability". Diese Initiative wird von internationalen Wissenschaftsorganisationen wie dem International Council for Science, dem International Social Science Council, und dem Belmont Forum (einem internationalen Netzwerk von Forschungsförderern, bei denen die Deutsche Forschungsgemeinschaft Mitglied ist) getragen. Armin Grunwald hat die Berufung angenommen.
\end{abstract}

$\ll \gg$

\section{Judith Simon gewinnt Herbert-A.-Simon-Preis}

Die ITAS-Kollegin Judith Simon wurde mit dem Herbert-A.-Simon-Preis 2013 ausgezeichnet, der jährlich von der International Association for Computing and Philosophy (IACAP) für außergewöhnliche Leistungen im Bereich „Philosophy $\&$ Computing" vergeben wird. Der Preis wird auf der diesjährigen IACAP-Tagung überreicht werden, die vom 15. bis 17. Juli 2013 in Maryland stattfinden wird.

Der Forschungsschwerpunkt von Judith Simon im ITAS ist die Analyse der epistemolo- 
gischen und ethischen Aspekte von IuK-Technologien. Sie leitet zudem ein Austrian-ScienceFund-Projekt zum Thema „Epistemic Trust in Socio-Technical Epistemic Systems“ an der Universität Wien und ist assoziiert am Institut Jean Nicod (Ecole normale supérieure) in Paris.

\section{$《 》$}

\section{Land Baden-Württemberg för- dert Schule der Nachhaltigkeit}

Die von ITAS verantwortete KIT-Einrichtung „Schule der Nachhaltigkeit" ist vom Wissenschaftsministerium Baden-Württemberg zur Förderung ausgewählt worden. Die Schule bietet fächerübergreifende Lehrmodule für Studenten an und baut damit einerseits auf den hervorragenden Kompetenzen des KIT in naturwissenschaftlichen und technischen Disziplinen auf. Andererseits fördert sie Reflexion, Urteilskraft, Gestaltungsfähigkeit und Verantwortungsbewusstsein im Sinne der Nachhaltigkeit. Die Förderung im Rahmen des Programms „Stärkung des Beitrags der Wissenschaft für eine nachhaltige Entwicklung“ beträgt rund 400.000 Euro für drei Jahre.

\section{Kontakt}

Dr. Oliver Parodi, ITAS

Tel.: +49 (0) 721 / 608 - 6816

E-Mail: oliver.parodi@kit.edu

\section{$《 》$}

\section{Personalia}

Daniel Ketzer ist seit April 2013 wissenschaftlicher Mitarbeiter im Forschungsbereich Umwelt und Nachhaltigkeit im Projekt „BioEnNW - Delivering Local Bioenergy to NW Europe“ am ITAS. Er absolvierte sein Bachelorstudium in Physischer Geographie in München und sein Masterstudium in Environmental Management and Physical Planning in Stockholm. Seine Arbeitsschwerpunkte sind Modellierung und GISbasierte Potentialanalysen für Bioenergie, Ag- rovoltaik und Photovoltaik sowie Bioökonomie. In seinem Promotionsprojekt mit der Universität Stockholm modelliert er Agrovoltaik-Potentiale, um Flächennutzungskonflikte zwischen Energieund Nahrungsmittelproduktion zu reduzieren.

Dominik Poncette ist seit Februar 2013 wissenschaftlicher Mitarbeiter im Forschungsbereich „Energie“ des ITAS und ist in das Industrieprojekt „Systemanalyse Windkraft-Wasserstoff“" sowie in die Helmholtz-Allianz „Energieeffiziente chemische Mehrphasenprozesse" und ein Helmholtz-Projekt der Energiespeicher- und Wasserstoffinitiative (EWI) zur chemischen Wärmespeicherung eingebunden. Sein Bachelorund Masterstudium absolvierte er an der RuhrUniversität Bochum im Fach „Sales Engineering \& Product Management" mit dem Schwerpunkt Energie- und Verfahrenstechnik.

Christa Streitferdt ist seit Januar 2013 als Projektassistentin für die Projektgruppen „Biomasse" und „Elektrische Speichersysteme" tätig. Nach einem Studium der Betriebswirtschaftslehre an der Fachhochschule Pforzheim betreute sie mehrere Jahre wissenschaftliche Forschungsprojekte als Projektkoordinatorin.

Patrick Sumpf ist seit September 2012 wissenschaftlicher Mitarbeiter im Projekt „Systemische Risiken in Energieinfrastrukturen" der Helmholtz-Allianz ENERGY-TRANS am ITAS. Er studierte Politikwissenschaft und Soziologie in Bielefeld und Berlin. Seine Arbeitsschwerpunkte sind Vertrauen, Risiko und Governance im Energiesektor. Patrick Sumpf bearbeitet in seinem Promotionsprojekt das Thema „Energy Transition in Future Critical Infrastructures Trust in the Smart Grid2“.

Markus Winkelmann arbeitet seit Dezember 2012 als wissenschaftlicher Mitarbeiter im Projekt „CONCERTO Premium“ am ITAS. Er studierte Soziologie, Psychologie und Geschichte in Freiburg und Madison und war anschließend als wissenschaftlicher Mitarbeiter am European Institute for Energy Research (EIfER), beim Max Rubner Institut (MRI) sowie an der Interfakultären Koordinationsstelle für Allgemeine Ökologie an der Universität Bern tätig. 


\section{Neue Veröffentlichungen}

\section{Neuerscheinung: Responsible Nanobiotechnology}

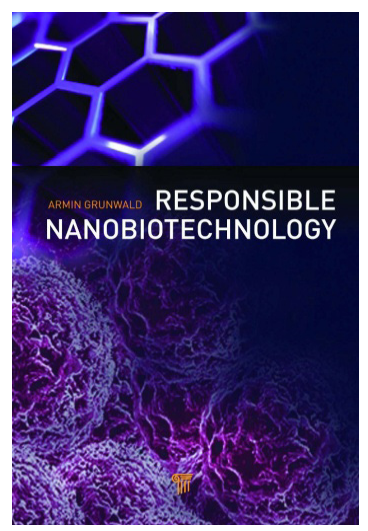

Hohe Erwartungen an die Nanotechnologie, aber auch bereits aufgetauchte und ebenso weitreichende Befürchtungen haben rasch ihre Tragweite für die gesellschaftliche Entwicklung der nächsten Jahrzehnte und die Zukunft des Menschen erkennen lassen. Das Buch nimmt die Überlegungen zum Thema „Ethik der Nanotechnologie" auf, die in der seit etwa zehn Jahren laufenden Debatte entwickelt wurden, systematisiert sie und entwickelt sie weiter. Der Schwerpunkt liegt auf den Forschungs- und Einsatzgebieten der Nanotechnologie an den Schnittstellen zur Biotechnologie, wie es das Wort „Nanobiotechnologie“ im Titel ausdrückt. Schwerpunkte der Reflexion sind die Themen Nanomaterialien und Vorsorgeprinzip, Synthetische Biologie und künstliches Leben, die nanotechnologische ,Verbesserung" von Tieren sowie die ,technische Verbesserung" des Menschen mit einem Schwerpunkt zu Neuroimplantaten. Herausforderungen an Verantwortung bzw. Fragen nach der Verantwortbarkeit bilden dabei den Rahmen.

Im Ergebnis zeigt sich ein breites Spektrum von ethisch relevanten Fragen der Nanobiotechnologie, die von den sehr konkreten Herausforderungen im Umgang mit Nanomaterialien bis hin zu den philosophischen Fragen zum Verhältnis von Mensch und Tier sowie zur Zukunft der Natur des Menschen reichen.

Dabei geht es nur zum Teil unmittelbar um ethische Reflexion, also um Antworten auf in der Frage „Was tun?“ enthaltene normative Unsicherheiten. Vielmehr schiebt sich immer wieder die philosophisch-hermeneutische Dimension der angesprochenen ,großen“ Fragen in den Vordergrund. Selbstverständigungsdebatten und Kontroversen über atomaren Reduktionismus, über das Verhältnis von Mensch, Natur, Tier und Technik oder über eine Technisierung des Menschen bzw. seine „technische Verbesserung“ prägen das Feld und eröffnen philosophische Anschlussmöglichkeiten auch jenseits der Ethik.

Bibliografische Angaben: Grunwald, A.: Responsible Nanobiotechnology: Philosophy and Ethics. Singapur: Pan Stanford Publ., 2012, ISBN 9789814316804, 383 S., \$ 149,95

\section{Neuerscheinung: Anerkennung in erweiterter Arbeit}

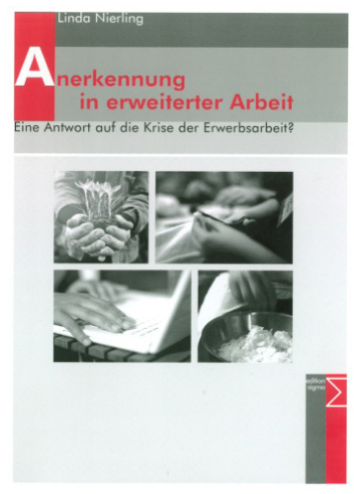

Dem deutschen Erwerbssystem werden seit Jahrzehnten immer wieder Krisen nachgesagt, und auch aktuell sind Krisenbefunde allgegenwärtig: Nicht nur prekäre Beschäftigungsverhältnisse, sondern auch die Zunahme beruflich bedingter Burnouts stehen im Fokus, während reproduktive Arbeitsfelder mehr und mehr an Bedeutung verlieren. Dieses Buch nimmt die Debatten um eine „Krise der Arbeitsgesellschaft“ der 1980er und 1990er Jahre zum Ausgang und arbeitet heraus, welchen Beitrag das damals entwickelte Konzept „erweiterter Arbeit“, das neben Erwerbsarbeit Arbeitsformen wie Familien-, Gemeinschaftsund Eigenarbeit integriert, zur Bewältigung dieser Krisenphänomene leisten kann.

Erweiterte Arbeitsformen bergen - so zeigt die Autorin - eine breite Vielfalt von „weichen“ 
Anerkennungserfahrungen. Sie ersetzen „harte", gesellschaftliche Anerkennungsstrukturen der Erwerbsarbeit nicht, doch können sie missachtende Erfahrungen aus anderen Bereichen kompensieren, die Subjekte bestärken und somit einen Beitrag zur Krisenbewältigung auf individueller Ebene darstellen.

Bibliografische Angaben: Nierling, L.: Anerkennung in erweiterter Arbeit. Eine Antwort auf die Krise der Erwerbsarbeit? Berlin: edition sigma, 2013, ISBN 978-3-8360-3591-0, 264 S., Euro 19,90

\section{Neuerscheinung: Alternative Antriebskon- zepte bei sich wandelnden Mobilitätsstilen}

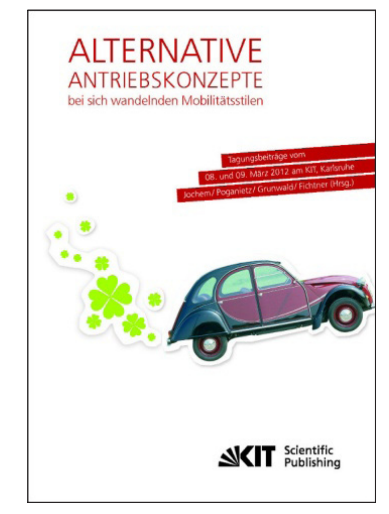

Kann die künftige Mobilität stärker von alternativen Antriebskonzepten geprägt sein? Werden sich die Mobilitätsgewohnheiten ändern und an die Antriebstechnologien anpassen? Und wie kann eine multimodale Mobilität aussehen?

Diese Fragestellung wurde auf einem KITWorkshop unter vier Aspekten nachgegangen: Entwicklung der individuellen Mobilität(-sstile), Stand und Perspektiven der Technik, Systemwirkungen sowie mögliche Zukünfte der Mobilität. Als Antriebstechnologien standen der Biokraftstoff nutzende konventionelle Verbrennungsmotor, der Elektromotor mit Brennstoffzelle sowie der Elektromotor mit Batterie im Fokus. Diese Alternativen haben, insbesondere auch vor dem Hintergrund der Energiewende, verschiedene Anforderungen zu erfüllen: Sie müssen technisch realisierbar und wirtschaftlich sein, ökologische und politisch-rechtliche Rahmenbedingungen erfüllen und nicht zuletzt gesellschaftlich akzeptiert werden. Ausgewählte Beiträge des Workshops sind in diesem Tagungsband veröffentlicht.

Bibliografische Angaben: Jochem, P.; Poganietz, W.R.; Grunwald, A.; Fichtner, W. (Hg.): Alternative Antriebskonzepte bei sich wandelnden Mobilitätsstilen: Tagungsbeiträge vom 8. und 9. März 2012 am KIT. Karlsruhe: KIT Scientific Publishing, 2013, ISBN 9783-86644-944-2, kostenloser Download unter http:// digbib.ubka.uni-karlsruhe.de/volltexte/1000031021

$\langle\gg$

\section{Neues Dissertationsprojekt}

\section{Konkrete Utopien einer offenen Technologie. Die Praktiken und Zukünfte des Open Design \\ von Christoph Schneider, ITAS}

„The future is open source everything.“ Dieser, im Internet viel zitierte Ausspruch, welcher den Erzählungen nach dem Gründer des Linux-Projektes in den Mund gelegt wird, trifft die Atmosphäre im Open-Source-Bereich der letzten Jahre. In einem horrenden Tempo diffundieren Open-SourcePraktiken, die ursprünglich aus der Softwareentwicklung stammen, inklusive ihrer normativen Ansprüche in viele gesellschaftliche Bereiche (Kelty 2008). „Open“ formiert sich zu einem heterogenen gesellschaftlichen Projekt, was exemplarisch an Organisationen wie der „Open Knowledge Foundation" sichtbar wird. Relativ jung sind Open-Source-Projekte, die mit der offenen und kooperativen Gestaltung und Nutzung von materiellen Artefakten experimentieren, im sog. „Open Design“ (teilweise auch „Open Source Hardware" genannt). Diese Sphäre steht im Zentrum meines soziologischen Promotionsvorhabens. In unterschiedlichsten Bereichen - von „Fenstergärten“ bis zu 3D-Druckern - entstehen neue Open-Source-Objekte und neue Open-SourceGemeinschaften. Diese arbeiten daran, bestimmte Relationen zwischen Menschen, Wissen und materiellen Objekten in ein offenes Paradigma zu transformieren, was u. a. bedeutet, Wissen zu teilen, verfügbar und veränderbar zu machen. Dies ändert nicht nur die Praktiken des Open Source, 
sondern erweitert auch die Vorstellungen davon, was künftig durch Open Source möglich und wünschenswert ist. Entsprechend analysiere ich Open-Design-Projekte als „Konkrete Utopien“ (Bloch 1959). Diese Perspektive impliziert nicht nur, dass Open-Design-Praktiken sich mit wünschenswerten Vorstellungen von Zukünften verbinden, sondern auch, dass Open-Design-Projekte selbst erklärungsbedürftig werden. Wie vermitteln diese bestehende Möglichkeiten und innovative Praktiken? Wie artikuliert sich das „Noch-Nicht“ (Bloch) im jetzt schon Geschehenden?

Daher betrachte ich Open-Design-Projekte nicht als isolierte Einheiten, sondern situiere sie innerhalb von "Assemblagen“ als im Prozess befindliche Cluster heterogener Elemente (DeLanda 2006). Open Design konstituiert und transformiert sich in der eingenommenen Perspektive erst durch das Zusammenspiel unterschiedlicher Einzelphänomene - wozu man z. B. Organisationen wie Creative Commons und das Fab Lab Netzwerk genauso zählen kann, wie die „Maker"-Bewegung oder Internet-Innovationen wie Crowdfunding. Allerdings ist die Art und Weise des Zusammenspiels von Open-DesignProjekten mit anderen Phänomenen eine Frage empirischer Forschung. Um den prozessualen und „konkret“ praktischen Aspekten des Open Design gerecht zu werden, fokussiert meine Arbeit empirisch auf die Objekte, die im Zentrum der Projekte stehen. Inspiriert ist dieser Ansatz etwa durch Konzepte der „Postsozialität“ (KnorrCetina 1997) und Perspektiven auf heterogene Mobilitäten (Urry 2007). Dass Objekte gemeinschaftsbildend wirken, und dabei komplexe Vermengungen der Bewegungen von Objekten, Wissen, Daten und Menschen stattfinden, ist zentral für Open Design. Daher legt die Nachverfolgung der Bewegungen von Open-Design-Objekten die Bedingungen der Möglichkeit dieser neuartigen Objekte offen und macht die Assemblagen transparent, an deren Transformation Open-DesignProjekte selbst teilhaben.

Letztendlich fragt das Promotionsprojekt danach, welche Visionen und Praktiken in Open Design entstehen und in welchem Zusammenhang diese mit einem breiteren gesellschaftlichen Projekt des Open Source stehen. Dabei rekonstruiere ich, welche neuen Formen des Wissens und der Objekte durch Open Design produziert werden. Konkrete Utopie dient dabei in der Arbeit als fruchtbare heuristische Perspektive, aber auch als Anspruch zur normativen Stellungnahme. Mein Promotionsprojekt versteht sich in gewisser Hinsicht auch als Teil des diffusen Gesellschaftsprojektes des Open Source. Reflexive soziologische Distanz wird aber dennoch eingehalten. Denn mit der Entstehung von Open Design ist nicht zugleich gesagt, dass die Zukunft „open source everything" sein wird, aber es treten neue Möglichkeiten in Erscheinung, die dazu beitragen, dass sie es sein kann. Diese soziologisch zu explorieren, ist das Ziel meiner Arbeit.

\section{Literatur}

Bloch, E., 1959: Das Prinzip Hoffnung. Frankfurt a. M. DeLanda, M., 2006: A New Philosophy of Society: Assemblage Theory and Social Complexity. London, New York

Kelty, Chr.M., 2008: Two Bits: The Cultural Significance of Free Software. Durham

Knorr-Cetina, K., 1997: Sociality with Objects: Social Relations in Postsocial Knowledge Societies. In: Theory, Culture \& Society, 14/4 (1997), S. 1-30

Urry, J., 2007: Mobilities. Cambridge

\section{Kontakt}

Christoph Schneider, ITAS

Tel.: +49 (0) 721 / 608 - 24792

E-Mail: christoph.schneider3@kit.edu 